\title{
Ranking candidate genes of esophageal squamous cell carcinomas based on differentially expressed genes and the topological properties of the co-expression network
}

Yuzhou Shen, Jicheng Tantai and Heng Zhao*

\begin{abstract}
Background: The aim of this study was to identify the candidate genes of esophageal squamous cell carcinoma (ESCC). Methods: Gene expression profiling of 17 ESCC samples and 17 adjacent normal samples, GSE20347, was downloaded from Gene Expression Omnibus database. The raw data were preprocessed, and the differentially expressed genes (DEGs) between ESCC and normal samples were identified by using SAM software (false discovery rate $<0.001$ ). Then, the co-expression network of DEGs was constructed based on Pearson's correlation test (r-value $\geq 0.8$ ). Furthermore, the topological properties of the co-expression network were analyzed through NetworkAnalyzer (default settings) of Cytoscape. The expression fold changes of DEGs and topological properties were utilized to identify the candidate genes of ESCC (Cri ${ }_{n}$ score $>4$ ), which were further analyzed based on DAVID functional enrichment analysis ( $P$-value $\left.<0.05\right)$.

Results: A total of 1,063 DEGs were identified, including 490 up-regulated and 573 down-regulated DEGs. Then, the co-expression network of DEGs was constructed, containing 999 nodes and 46,323 edges. Based on the expression fold changes of DEGs and the topological properties of the co-expression network, DEGs were ranked, and the top 24 genes were candidate genes of ESCC, such as CRISP3, EREG, CXCR2, and CRNN. Furthermore, the 24 genes were significantly enriched in bio-functions regarding cell differentiation, glucan biosynthetic process and immune response.

Conclusion: The present study suggested that CRISP3, EREG, CXCR2, and CRNN might be causative genes of ESCC, and play vital roles in the development of ESCC. However, further experimental studies are needed to confirm our results.

Keywords: Esophageal squamous cell carcinomas, Differentially expressed genes, Topological properties, Co-expression network, Candidate genes
\end{abstract}

\section{Background}

Esophageal squamous cell carcinoma (ESCC) is the predominant histologic subtype of esophageal cancer, which is characterized by high mortality rate and geographic differences in incidence [1]. ESCC is a common malignant cancer worldwide, especially in China [2]. Recently, clinical therapies have been used to treat ESCC, such as neoadjuvant chemoradiotherapy [3,4], surgery [5], and combination therapy [6]. However, these approaches do not increase survival rate of patients with ESCC. To

\footnotetext{
* Correspondence: hengzhao2233@hotmail.com

Department of Thoracic Surgery, Shanghai Chest Hospital, Shanghai Jiao Tong University, 241 Huaihai (West) Rd, Shanghai 200030, People's Republic of China
}

\section{Biomed Central}

investigate novel therapies, a clear understanding of the molecular pathogenesis of ESCC is required, to which much effort has been made.

Microarray analysis has been widely used to investigate gene expression in various diseases, such as breast tumors [7], brain cancer [8], endometrial cancer [9], and renal cell carcinoma [10]. In previous studies of ESCC, loss of heterozygosity (LOH) and copy number alteration in ESCC were identified by using microsatellite markers and low- and high-density SNP arrays $[11,12]$. The differentially expressed genes (DEGs) and microRNAs between ESCC and normal squamous epithelia have been identified based on microarray analysis $[13,14]$. Moreover, a comprehensive survey of commonly inactivated tumor suppressor genes 
in ESCC was performed based on microarray analysis and functional reactivation of silenced tumor suppressor genes by 5 -aza-2'-deoxycytidine and trichostatin [15]. Thus, microarray analysis is a useful approach to identify key genes involved in ESCC.

In our study, microarrays were utilized to identify the DEGs between human ESCC samples and adjacent normal tissues samples. Then, the co-expression network of DEGs was constructed, and the topological properties of the co-expression network were analyzed. Additionally, we built an integrated index to rank DEGs and identify the candidate genes of ESCC. Furthermore, the relevant functions of candidate genes were investigated. We anticipate that our work can find key genes related with ESCC, and provide new insights for target therapies.

\section{Methods}

\section{Microarray data and preprocessing}

The raw gene expression profile GSE20347 [2] was downloaded from the public functional genomics database Gene Expression Omnibus (GEO, http://www.ncbi.nlm. nih.gov/geo/). In total, 34 specimens were available, including 17 human ESCC samples and 17 matched adjacent normal tissues samples. None of the patients with ESCC had received prior therapy, and informed consent was obtained. Demographic and clinical information were matched. The corresponding platform was GPL571, [HG-U133A_2] Affymetrix Human Genome U133A 2.0 Array. The background correction and normalization of microarray data among chips were conducted based on RMA (Robust Multiarray Averaging) method [16]. When several probes were mapped to one gene, their mean value was taken as the gene expression value of this gene.

\section{DEG screening}

SAM 4.0 software [17] was used to screen out the DEGs between ESCC and normal samples. SAM is a widely used tool for DEG screening. The criterion for this analysis was false discovery rate (FDR) $<0.001$.

\section{Hierarchical clustering analysis of DEGs}

To determine the specificity of DEGs between ESCC and adjacent normal samples, the pheatmap package in $\mathrm{R}$ was utilized to perform bidirectional hierarchical clustering analysis (BHCA) [18,19]. Rationally, after BHCA, ESCC and adjacent normal samples are supposed to be distinguished clearly by DEGs.

\section{Constructing the co-expression network of DEGs}

In organisms, biological functions are often based on the interaction of several genes, and significantly co-expressed genes are usually co-regulated and involved in the same or similar biological processes and pathways [20]. In order to identify the co-expressed DEGs in ESCC tissues, the expression values of DEGs in each sample were abstracted, and Pearson's correlation test was used to calculate the correlation coefficient ( $r$-value) of DEGs. Higher $P$-value represents stronger correlation between node ' $n$ ' and its adjacent nodes, and only the co-expressed DEG pairs with r-value $\geq 0.8$ were utilized to construct the co-expression network of DEGs, which was visualized by using Cytoscape [21].

\section{Analyzing the topological properties of the co-expression network}

The topological properties (node degree and clustering coefficient) of DEGs in the co-expression network were analyzed based on NetworkAnalyzer of Cytoscape software [22]. Node degree and clustering coefficient are important topological properties of network. Node degree ' $k_{n}$ ' is the number of nodes connected to node 'n', displaying the local centrality of this node in network. Higher node degree represents stronger importance of a node for the stability of network. Clustering coefficient of node ' $n$ ' is defined as:

$$
C_{n}=2 e_{n} /\left[k_{n}\left(k_{n}-1\right)\right]
$$

In this formula, ' $k_{n}$ ' is the number of adjacent nodes, and ' $e_{n}$ ' is the number of interconnections among adjacent nodes. Representing the clustering degree of node ' $n$ ', $C_{n}$ is between 0 and 1 , and higher $C_{n}$ represents that the adjacent nodes of node ' $n$ ' connected with each other more closely.

\section{DEG ranking and candidate gene identification}

Candidate genes of ESCC could be identified by ranking DEGs based on expression fold changes, node degrees, and clustering coefficients in the co-expression network. In this study, firstly, Z-transformation was performed to transform three sets of data into Z-scores, which are common standardized scores in statistics [23]. Secondly, the ranking index of each DEG was calculated based on the formula as follows:

$$
\mathrm{Cri}_{\mathrm{n}}=\mathrm{FC}_{\mathrm{n}}+\text { degree }_{\mathrm{n}}+\mathrm{C}
$$

Here, $\mathrm{Cri}_{n}$ is the ranking index of gene ' $\mathrm{n}$ ', $\mathrm{FC}_{\mathrm{n}}$ is the expression fold change of gene 'n', degree $_{n}$ is the node degree of gene ' $n$ ' in theco-expression network, and $C_{n}$ is the clustering coefficient of gene ' $n$ ' in the co-expression network. Thirdly, the DEGs with $\mathrm{Cri}_{n}$ score $>4$ were defined as candidate genes of ESCC.

\section{Functional enrichment analysis}

To reveal the biological process associated with ESCC, gene ontology (GO) functional enrichment analysis was performed for the candidate genes of ESCC based on 
the Database for Annotation, Visualization and Integrated Discovery (DAVID) [24]. DAVID provides exploratory visualization tools to promote functional classification. In this study, the criterion for this analysis was a $P$ value $<0.05$.

\section{Results}

Identification of DEGs and hierarchical clustering analysis After preprocessing and DEG screening, 1,063 DEGs (FDR $<0.001$ ) were identified between ESCC and adjacent normal samples, including 490 up-regulated and 573 down-regulated DEGs. After BHCA, ESCC and adjacent normal samples could be distinguished clearly by DEGs (Figure 1).

\section{Construction of the co-expression network}

The $r$-values of DEG pairs were calculated based on Pearson's correlation test, and the co-expression network of DEGs with $r$-value $\geq 0.8$ were constructed (Additional file 1). The co-expression network involved 999 nodes (DEGs) and 46,323 edges (co-expression relationships),

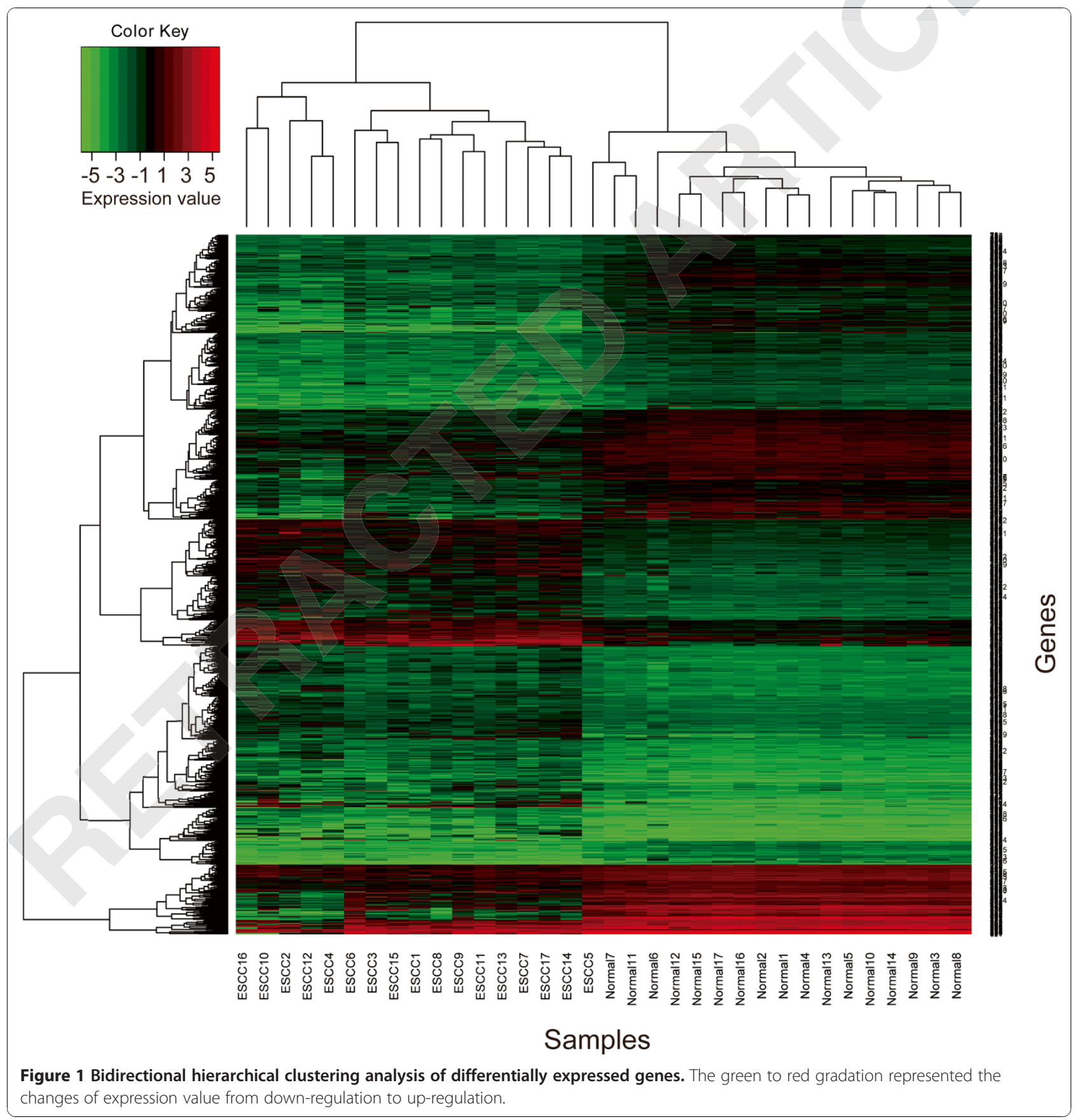


and it was further divided into two closely connected large sub-networks.

\section{DEG ranking and candidate gene identification}

To identify the candidate genes of ESCC, we established an integrated ranking index by integrating the expression fold changes, node degrees, and clustering coefficients of DEGs in the co-expression network. Consequently, a total of 24 genes were candidate genes $\left(\mathrm{Cri}_{\mathrm{n}}\right.$ score $\left.>4\right)$ of ESCC (Table 1), such as cysteine-rich secretory protein 3 (CRISP3), epiregulin (EREG), chemokine receptor 2 (CXCR2), and cornulin (CRNN).

\section{Functional enrichment analysis}

To understand the biological processes involving candidate genes, GO functional enrichment analysis $(P$-value $<0.05)$ was performed. It was found that the 24 candidate genes were significantly enriched in bio-functions regarding cell differentiation, glucan biosynthetic process and immune response, including epidermal cell differentiation, epithelial cell differentiation, epidermis development, keratinocyte differentiation, glucan biosynthetic process, and regulation of immune response (Table 2).

\section{Discussion}

In the present study, we identified 1,063 DEGs between ESCC and adjacent normal samples, including 490 upregulated and 573 down-regulated genes. Furthermore, the co-expression network of DEGs was constructed, consisting of 2 large sub-networks, 999 nodes, and 46,323 edges. Then, the expression fold changes, node degrees, and clustering coefficients of DEGs in the co-expression network were comprehensively analyzed to rank DEGs, and 24 candidate genes of ESCC were identified.

Among the 24 candidate genes, the highest ranking was CRISP3. It was originally discovered in human

Table 1 The $\mathbf{2 4}$ candidate genes of esophageal squamous cell carcinomas

\begin{tabular}{llll}
\hline Rank & Symbol & Rank & Symbol \\
\hline 1 & CRISP3 & 13 & CYP4B1 \\
2 & EREG & 14 & SLURP1 \\
3 & ENDOU & 15 & MAL \\
4 & CXCR2 & 16 & TGM3 \\
5 & CRCT1 & 17 & CWH43 \\
6 & FLG & 18 & TMPRSSIIE \\
7 & CLIC3 & 19 & CLCA4 \\
8 & CRNN & 20 & PPPIR3C \\
9 & MMP1 & 21 & BBOX1 \\
10 & GYS2 & 22 & HPGD \\
11 & EPB4TL3 & 23 & SPINK5 \\
12 & SCEL & 24 & FCERTA \\
\hline
\end{tabular}

Table 2 Functional enrichment analysis of the 24 candidate genes

\begin{tabular}{lll}
\hline GO ID & GO term & P-value \\
\hline GO:0009913 & Epidermal cell differentiation & $2.43 \mathrm{E}-06$ \\
GO:0030855 & Epithelial cell differentiation & $3.14 \mathrm{E}-05$ \\
GO:0008544 & Epidermis development & $9.93 \mathrm{E}-05$ \\
GO:0030216 & Keratinocyte differentiation & $1.10 \mathrm{E}-04$ \\
GO:0008544 & Epidermis development & $1.21 \mathrm{E}-04$ \\
GO:0007398 & Ectoderm development & $1.34 \mathrm{E}-04$ \\
GO:0060429 & Epithelium development & $2.23 \mathrm{E}-04$ \\
GO:0009888 & Tissue development & $1.15 \mathrm{E}-02$ \\
GO:0009250 & Glucan biosynthetic process & $1.70 \mathrm{E}-02$ \\
GO:0005978 & Glycogen biosynthetic process & $1.70 \mathrm{E}-02$ \\
GO:0033692 & Cellular polysaccharide biosynthetic process & $3.54 \mathrm{E}-02$ \\
GO:0050776 & Regulation of immune response & $4.14 \mathrm{E}-02$ \\
GO:0044042 & Glucan metabolic process & $4.87 \mathrm{E}-02$ \\
GO:0006073 & Cellular glucan metabolic process & $4.87 \mathrm{E}-02$ \\
GO:0009913 & Epidermal cell differentiation & $2.43 \mathrm{E}-06$ \\
GO:0030855 & Epithelial cell differentiation & $3.14 \mathrm{E}-05$ \\
\hline GO: & &
\end{tabular}

GO: gene ontology; ID: identifier.

neutrophilic granulocytes, and is a glycoprotein that belongs to a family of cysteine-rich secretory proteins (CRISPs) [25]. In previous studies, CRISP3 is found to be overexpressed in prostate adenocarcinoma by using quantitative real-time reverse-transcription-PCR [26]. Additionally, Su et al. reveal that CRISP3 is significantly down-regulated in ESCC, and may be the biomarker of ESCC [27]. Furthermore, CRISP3 was reported to be down-regulated in oral squamous cell carcinoma (OSCC), and the loss of its DNA copy number was observed in two of the five OSCC-derived cell lines [28].

EREG was the second highest ranking of 24 candidate genes whose protein product, EREG, induces cell growth by binding to the epidermal growth factor receptor (EGFR) [29]. It is reported that EREG is epigenetically silenced in gastric cancer cells by aberrant DNA methylation and histone modification [29]. Moreover, EREG is involved in the invasion and metastasis of esophageal carcinoma by combining with sphingosine kinase-1 (SPHK1) [30].

CXCR2I codes a receptor of ELR + CXC chemokines, which are potent promoters of angiogenesis [31]. It is reported that GROA-C XCR2 and GROB-C XCR2 signaling contribute significantly to esophageal cancer cell proliferation, and this autocrine signaling pathway may be involved in esophageal tumorigenesis [32,33].

CRNN codes cornulin, a $\mathrm{Ca}^{2+}$ - binding protein that presents in the upper layer of squamous epithelia [34]. It has been shown that the large majority of ESCC cases have little or no expression of cornulin in carcinoma or stromal cells [35]. These evidences suggested that CRISP3, 
EREG, CXCR2, and CRNN may play crucial roles in ESCC, as well as other candidate genes.

In addition, GO functional enrichment analysis was performed, and some biological processes were enriched significantly, such as epidermal cell differentiation, epithelial cell differentiation, epidermis development, keratinocyte differentiation, and regulation of the immune response. It has been shown that proliferation and development of esophageal epithelial cells are associated with the development of ESCC [36]. Moreover, ESCC-related gene modules are significantly enriched in epidermal cell differentiation, epithelial cell differentiation, epidermis development, and keratinocyte differentiation [37]. Additionally, keratinocytes migrate from the basal to the superficial layers of the epidermis, and undergo morphological and biochemical changes during terminal differentiation, which are involved in the development of ESCC [38,39]. Our results were consistent with these evidences.

\section{Conclusions}

In conclusion, the DEGs between ESCC and adjacent normal tissues were screened out, and the co-expression network was constructed, consisting of 2 large subnetworks, 999 nodes, and 46,323 edges. After analyzing the gene expression and topological properties of DEGs in the co-expression network, DEGs were ranked, and 24 candidate genes of ESCC were identified. Candidate genes, such as CRISP3, EREG, CXCR2, and CRNN, were identified as potentially playing key roles in the development of ESCC. Furthermore, functional enrichment analysis revealed that the 24 genes were mainly enriched in epithelial cell differentiation, epidermis development, and keratinocyte differentiation. These results provided us with candidate genes and demonstrated their potential functions in the development of ESCC. However, more experimental studies are needed to confirm these results.

\section{Additional file}

\section{Additional file 1: The co-expression network of differentially} expressed genes.

\section{Competing interests}

The authors declare that they have no competing interests.

\section{Authors' contributions}

YS carried out the design and coordinated the study, participated in most of the experiments and prepared the manuscript. JT provided assistance in the design of the study, coordinated and carried out all the experiments and participated in manuscript preparation. $\mathrm{HZ}$ provided assistance for all experiments. All authors have read and approved the content of the manuscript.

Authors' information

Yuzhou Shen and Jicheng Tantai are joint first authors.

\section{Acknowledgements}

The author is grateful to the members of Department of Thoracic Surgery, Shanghai Chest Hospital affiliated to Shanghai Jiao Tong University, for their highly valued laboratory assistance.

Received: 23 March 2014 Accepted: 22 September 2014 1.

\section{References}

1. Lam AKY: Molecular biology of esophageal squamous cell carcinoma. Crit Rev Oncol Hematol 2000, 33:71-90.

2. Hu N, Clifford R, Yang H, Wang C, Goldstein A, Ding T, Taylor P, Lee M: Genome wide analysis of DNA copy number neutral loss of heterozygosity (CNNLOH) and its relation to gene expression in esophageal squamous cell carcinoma. BMC Genomics 2010, 11:576.

3. Wieder HA, Brücher BL, Zimmermann F, Becker K, Lordick F, Beer A, Schwaiger M, Fink U, Siewert JR, Stein HJ: Time course of tumor metabolic activity during chemoradiotherapy of esophageal squamous cell carcinoma and response to treatment. J Clin Oncol 2004, 22:900-908.

4. Brücher BL, Weber W, Bauer M, Fink U, Avril N, Stein HJ, Werner M, Zimmerman F, Siewert JR, Schwaiger M: Neoadjuvant therapy of esophageal squamous cell carcinoma: response evaluation by positron emission tomography. Ann Surg 2001, 233:300.

5. Stahl M, Stuschke M, Lehmann N, Meyer H-J, Walz MK, Seeber S, Klump B, Budach W, Teichmann R, Schmitt M: Chemoradiation with and without surgery in patients with locally advanced squamous cell carcinoma of the esophagus. J Clin Oncol 2005, 23:2310-2317.

6. Tepper J, Krasna MJ, Niedzwiecki D, Hollis D, Reed CE, Goldberg R, Kiel K, Willett C, Sugarbaker D, Mayer R: Phase III trial of trimodality therapy with cisplatin, fluorouracil, radiotherapy, and surgery compared with surgery alone for esophageal cancer: CALGB 9781. J Clin Oncol 2008, 26:1086-1092. Pollack JR, Sørlie T, Perou CM, Rees CA, Jeffrey SS, Lonning PE, Tibshirani R, Botstein D, Børresen-Dale A-L, Brown PO: Microarray analysis reveals a major direct role of DNA copy number alteration in the transcriptional program of human breast tumors. Proc Natl Acad Sci 2002, 99:12963-12968.

8. Mischel PS, Cloughesy TF, Nelson SF: DNA-microarray analysis of brain cancer: molecular classification for therapy. Nat Rev Neurosci 2004, 5:782-792.

9. Risinger Jl, Maxwell GL, Chandramouli G, Jazaeri A, Aprelikova O, Patterson T, Berchuck A, Barrett JC: Microarray analysis reveals distinct gene expression profiles among different histologic types of endometrial cancer. Cancer Res 2003, 63:6-11.

10. Moch H, Schraml P, Bubendorf L, Mirlacher M, Kononen J, Gasser T, Mihatsch MJ, Kallioniemi OP, Sauter G: High-throughput tissue microarray analysis to evaluate genes uncovered by cDNA microarray screening in renal cell carcinoma. Am J Pathol 1999, 154:981-986.

11. Hu N, Roth MJ, Emmert-Buck MR, Tang Z-Z, Polymeropolous M, Wang Q-H Goldstein AM, Han X-Y, Dawsey SM, Ding T, Giffen C, Taylor PR: Allelic loss in esophageal squamous cell carcinoma patients with and without family history of upper gastrointestinal tract cancer. Clin Cancer Res 1999, 5:3476-3482.

12. Hu N, Wang C, Hu Y, Yang HH, Kong L-H, Lu N, Su H, Wang Q-H, Goldstein AM, Buetow KH: Genome-wide loss of heterozygosity and copy number alteration in esophageal squamous cell carcinoma using the Affymetrix GeneChip mapping $10 \mathrm{~K}$ array. BMC Genomics 2006, 7:299.

13. Luo A, Kong J, Hu G, Liew C-C, Xiong M, Wang X, Ji J, Wang T, Zhi H, Wu M: Discovery of $\mathrm{Ca}^{2+}$-relevant and differentiation-associated genes downregulated in esophageal squamous cell carcinoma using cDNA microarray. Oncogene 2003, 23:1291-1299.

14. Kimura S, Naganuma S, Susuki D, Hirono Y, Yamaguchi A, Fujieda S, Sano K, Itoh $\mathrm{H}$ : Expression of microRNAs in squamous cell carcinoma of human head and neck and the esophagus: miR-205 and miR-21 are specific markers for HNSCC and ESCC. Oncol Rep 2010, 23:1625-1633.

15. Yamashita K, Upadhyay S, Osada M, Hoque MO, Xiao Y, Mori M, Sato F, Meltzer SJ, Sidransky D: Pharmacologic unmasking of epigenetically silenced tumor suppressor genes in esophageal squamous cell carcinoma. Cancer Cell 2002, 2:485-495.

16. Irizarry RA, Hobbs B, Collin F, Beazer-Barclay YD, Antonellis KJ, Scherf U, Speed TP: Exploration, normalization, and summaries of high density oligonucleotide array probe level data. Biostatistics 2003, 4:249-264. 
17. Tusher VG, Tibshirani R, Chu G: Significance analysis of microarrays applied to the ionizing radiation response. Proc Natl Acad Sci 2001 98:5116-5121

18. Deza M, Deza E: Encyclopedia of Distances. In Encyclopedia of Distances. Berlin Heidelberg: Springer; 2009:94.

19. Szekely GJ, Rizzo ML: Hierarchical clustering via joint between-within distances: extending Ward's minimum variance method. J Classif 2005, 22:151-183.

20. Allocco DJ, Kohane IS, Butte AJ: Quantifying the relationship between co-expression, co-regulation and gene function. BMC Bioinformatics 2004 $5: 18$.

21. Shannon P, Markiel A, Ozier O, Baliga NS, Wang JT, Ramage D, Amin N, Schwikowski B, Ideker T: Cytoscape: a software environment for integrated models of biomolecular interaction networks. Genome Res 2003, 13:2498-2504.

22. Assenov Y, Ramirez F, Schelhorn SE, Lengauer T, Albrecht M: Computing topological parameters of biological networks. Bioinformatics 2008 24:282-284.

23. Quackenbush J: Microarray data normalization and transformation. Nat Genet 2002, 32:496-501.

24. Sherman BT, Lempicki RA: Bioinformatics enrichment tools: paths toward the comprehensive functional analysis of large gene lists. Nucleic Acids Res 2009, 37:1-13.

25. Udby L, Cowland JB, Johnsen AH, Sørensen OE, Borregaard N, Kjeldsen L: An ELISA for SGP28/CRISP-3, a cysteine-rich secretory protein in human neutrophils, plasma, and exocrine secretions. J Immunol Methods 2002, 263:43-55.

26. Kosari F, Asmann YW, Cheville JC, Vasmatzis G: Cysteine-rich secretory protein-3: a potential biomarker for prostate cancer. Cancer Epidemiol Biomark Prev 2002, 11:1419-1426.

27. Su H, Hu N, Yang HH, Wang C, Takikita M, Wang Q-H, Giffen C, Clifford R, Hewitt SM, Shou J-Z, Goldstein AM, Lee MP, Taylor PR: Global gene expression profiling and validation in esophageal squamous cell carcinoma and its association with clinical phenotypes. Clin Cancer Res 2011, 17:2955-2966

28. Ko W-C, Sugahara K, Sakuma T, Yen C-Y, Liu S-Y, Liaw G-A, Shibahara T: Copy number changes of CRISP3 in oral squamous cell carcinoma. Oncol Lett 2012, 3:75-81.

29. Yun J, Song S-H, Park J, Kim H-P, Yoon Y-K, Lee K-H, Han S-W, Oh D-Y, Im S-A, Bang Y-J: Gene silencing of EREG mediated by DNA methylation and histone modification in human gastric cancers. Lab Investig 2012, 92:1033-1044.

30. Pan J, Tao Y-F, Zhou Z, Cao B, Wu S-Y, Zhang Y-L, Hu S-Y, Zhao W-L, Wang J, Lou G-L: An novel role of sphingosine kinase-1 (SPHK1) in the invasion and metastasis of esophageal carcinoma. J Trans/ Med 2011, 9:157.

31. Belperio JA, Keane MP, Arenberg DA, Addison CL, Ehlert JE, Burdick MD, Strieter RM: CXC chemokines in angiogenesis. J Leukoc Biol 2000, 68:1-8.

32. Wang B, Hendricks DT, Wamunyokoli F, Parker MI: A growth-related oncogene/CXC chemokine receptor 2 autocrine loop contributes to cellular proliferation in esophageal cancer. Cancer Res 2006 66:3071-3077.

33. Wang B, Khachigian LM, Esau L, Birrer MJ, Zhao X, Parker MI, Hendricks DT: A key role for early growth response- 1 and nuclear factor-kB in mediating and maintaining GRO/CXCR2 proliferative signaling in esophageal cancer. Mol Cancer Res 2009, 7:755-764

34. Contzler R, Favre B, Huber M, Hohl D: Cornulin, a new member of the 'fused gene' family, is expressed during epidermal differentiation. J Investig Dermatol 2005, 124:990-997.

35. Pawar H, Maharudraiah J, Kashyap MK, Sharma J, Srikanth SM, Choudhary R, Chavan S, Sathe G, Manju HC, Kumar KV, Vijayakumar M, Sirdeshmukh R, Harsha HC, Prasad TS, Pandey A, Kumar RV: Downregulation of cornulin in esophageal squamous cell carcinoma. Acta Histochem 2013, 115:89-99.

36. Zhang H, Chen W, Duan C-J, Zhang C-F: Overexpression of HSPA2 is correlated with poor prognosis in esophageal squamous cell carcinoma. World J Surg Oncol 2013, 11:141.

37. Gao H, Wang L, Cui S, Wang M: Combination of meta-analysis and graph clustering to identify prognostic markers of ESCC. Genet Mol Biol 2012 35:530-537.
38. Kadowaki Y, Fujiwara T, Fukazawa T, Shao J, Yasuda T, Itoshima T, Kagawa S, Hudson LG, Roth JA, Tanaka N: Induction of differentiation-dependent apoptosis in human esophageal squamous cell carcinoma by adenovirus-mediated p21sdi1 gene transfer. Clin Cancer Res 1999, 5:4233-4241

39. Banks-Schlegel SP, Quintero J: Growth and differentiation of human esophageal carcinoma cell lines. Cancer Res 1986, 46:250-258.

\section{doi:10.1186/s40001-014-0052-x}

Cite this article as: Shen et al:: Ranking candidate genes of esophageal squamous cell carcinomas based on differentially expressed genes and the topological properties of the co-expression network. European Journal of Medical Research 2014 19:52

\section{Submit your next manuscript to BioMed Central and take full advantage of:}

- Convenient online submission

- Thorough peer review

- No space constraints or color figure charges

- Immediate publication on acceptance

- Inclusion in PubMed, CAS, Scopus and Google Scholar

- Research which is freely available for redistribution 